Article

\title{
The Approximation Property of a One-Dimensional, Time Independent Schrödinger Equation with a Hyperbolic Potential Well
}

\author{
Ginkyu Choi ${ }^{1,+}$ and Soon-Mo Jung ${ }^{2, *,+}$ (i) \\ 1 Department of Electronic and Electrical Engineering, College of Science and Technology, Hongik University, \\ Sejong 30016, Korea; gkchoi@hongik.ac.kr \\ 2 Mathematics Section, College of Science and Technology, Hongik University, Sejong 30016, Korea \\ * Correspondence: smjung@hongik.ac.kr; Tel.: +82-44-860-2584 \\ $\dagger$ These authors contributed equally to this work.
}

Received: 8 July 2020; Accepted: 10 August 2020; Published: 12 August 2020

\begin{abstract}
A type of Hyers-Ulam stability of the one-dimensional, time independent Schrödinger equation was recently investigated; the relevant system had a parabolic potential wall. As a continuation, we proved a type of Hyers-Ulam stability of the time independent Schrödinger equation under the action of a specific hyperbolic potential well. One of the advantages of this paper is that it proves a type of Hyers-Ulam stability of the Schrödinger equation under the condition that the potential function has singularities.
\end{abstract}

Keywords: generalized Hyers-Ulam stability; Hyers-Ulam stability; Schrödinger equation; time independent Schrödinger equation; hyperbolic potential well

\section{Introduction}

About 80 years ago, S. M. Ulam [1] discussed several important unsolved problems in a mathematics club at the University of Wisconsin. Among them was a question about the Hyers-Ulam stability of group homomorphisms:

Assume that $G_{1}$ is a group and $G_{2}$ is a metric group with the metric $d(\cdot, \cdot)$. For any given $\delta>0$,

does there exist a $\varepsilon>0$ such that if a function $h: G_{1} \rightarrow G_{2}$ satisfies $d(h(x y), h(x) h(y))<\varepsilon$ for all

$x, y \in G_{1}$, then there exists a homomorphism $H: G_{1} \rightarrow G_{2}$ with $d(h(x), H(x))<\delta$ for all $x \in G_{1}$ ?

In 1941, the following year, D. H. Hyers [2] was able to partially solve the Ulam's question for approximately additive functions, assuming that $G_{1}$ and $G_{2}$ were Banach spaces. Indeed, he proved that every solution to the inequality $\|f(x+y)-f(x)-f(y)\| \leq \varepsilon$ (for all $x$ and $y$ ) can be approximated by an exact solution (additive function). In this case, the Cauchy additive functional equation, $f(x+y)=f(x)+f(y)$, is said to have (or satisfy) the Hyers-Ulam stability.

Meanwhile, Th. M. Rassias [3] attempted to weaken the condition imposed on the upper bound for the "size" of Cauchy differences as follows:

$$
\|f(x+y)-f(x)-f(y)\| \leq \varepsilon\left(\|x\|^{p}+\|y\|^{p}\right)
$$

and he proved the Hyers' theorem, when he assumed that $p$ was a nonnegative real constant but $p \neq 1$. In other words, he proved the Hyers-Ulam-Rassias stability of the Cauchy additive functional equation. Since then, P. Găvruţa [4] published a paper that further generalized the theorem of Rassias, and these two papers have been enough to attract the attention of many mathematicians (see [5-7]). 
Given an open interval $I=(a, b)$ with $-\infty \leq a<b \leq+\infty$ and an $n \in \mathbb{N}$, we will define the Hyers-Ulam stability of the $n$ th-order linear differential equation

$$
a_{n}(x) y^{(n)}(x)+a_{n-1}(x) y^{(n-1)}(x)+\cdots+a_{1}(x) y^{\prime}(x)+a_{0}(x) y(x)=g(x),
$$

where the function $y: I \rightarrow \mathbb{C}$ is assumed to be $n$ times continuously differentiable and the coefficient functions $a_{i}: I \rightarrow \mathbb{C}$ are continuous, and where the inhomogeneous term $g: I \rightarrow \mathbb{C}$ is also assumed to be continuous.

The linear differential Equation (1) is said to have (or satisfy) the Hyers-Ulam stability if and only if the following statement holds true for any given $\varepsilon>0$ : For every $n$ times continuously differentiable function $y: I \rightarrow \mathbb{C}$ satisfying the differential inequality

$$
\left|a_{n}(x) y^{(n)}(x)+a_{n-1}(x) y^{(n-1)}(x)+\cdots+a_{1}(x) y^{\prime}(x)+a_{0}(x) y(x)-g(x)\right| \leq \varepsilon
$$

for all $x \in I$, the differential Equation (1) has a solution $y_{0}: I \rightarrow \mathbb{C}$ with

$$
\left|y(x)-y_{0}(x)\right| \leq K_{\varepsilon}(x)
$$

for every $x \in I$, where $K_{\varepsilon}(x)$ is dependent on $x$ and $\varepsilon$ and $\lim _{\varepsilon \rightarrow 0} K_{\varepsilon}(x)=0$ regardless of values of $x$.

Now we consider the case when $\sup _{x \in I} \lim _{\varepsilon \rightarrow 0} K_{\varepsilon}(x)>0$. As this phenomenon seems slightly different from the Hyers-Ulam stability in a broad sense, we should say that the differential Equation (1) has a type of Hyers-Ulam stability. We advise interested readers to refer to [5-7] for a detailed definition of Hyers-Ulam stability.

As far as we know, M. Obłoza [8,9] was the first person to prove the Hyers-Ulam stability of differential equations. Indeed, Obłoza dealt with Hyers-Ulam stability of the linear differential equation $y^{\prime}(x)+f(x) y(x)=g(x)$. Since then, many mathematicians have become interested in this subject and they have dealt with it more broadly and in depth (see [3,10-20]).

In a recent paper [21], the one-dimensional, time independent Schrödinger equation

$$
-\frac{\hbar^{2}}{2 m} \frac{d^{2} \psi(x)}{d x^{2}}+V(x) \psi(x)=E \psi(x)
$$

was considered, and a type of Hyers-Ulam stability was investigated; the system under observation had a parabolic potential wall.

In the present paper, we deal with the one-dimensional, time independent Schrödinger Equation (2), where $\psi:(0, c) \rightarrow \mathbb{C}$ is the wave function, $V$ is a hyperbolic potential well, $\hbar=\frac{h}{2 \pi}$ is the reduced Planck constant, $m$ is the mass of the particle, and $E$ is the energy level of the system. Indeed we investigate a type of Hyers-Ulam stability of the Schrödinger Equation (2) under the action of hyperbolic potential well with a singularity point at $x=0$ and $0<E<V_{0}$.

Finally, we need to mention that we wrote this paper using the ideas and experience of the papers $[16,18,21,22]$.

\section{Preliminaries}

The explicit formula for the general solution to the first-order linear differential equation is well known. We briefly recall that formula in the following lemma.

Lemma 1. Suppose $I=(0, c)$ is an open interval and $c>0$ is a fixed real number. Assume moreover that the functions $f, g: I \rightarrow \mathbb{C}$ are continuous such that every integral below exists. Then each continuously differentiable function $y: I \rightarrow \mathbb{C}$ is a solution of the first-order linear inhomogeneous differential equation

$$
y^{\prime}(x)+f(x) y(x)=g(x)
$$


if and only if $y$ is expressed by the formula

$$
y(x)=\exp \left(\int_{x}^{c} f(w) d w\right)\left(y(c)-\int_{x}^{c} g(s) \exp \left(-\int_{s}^{c} f(w) d w\right) d s\right),
$$

where $y(c)$ is a complex number.

Using Lemma 1, we can easily prove the generalized Hyers-Ulam stability of the linear, inhomogeneous differential equation of first order. This theorem is already well known, so readers interested in reading its proof may refer to the paper [21]. In the following lemma, $\Re(z)$ denotes the real part of the complex number $z$.

Lemma 2. Suppose $I=(0, c)$ is an open interval for a fixed real number $c>0$. Assume that the functions $f, g: I \rightarrow \mathbb{C}$ and $\varphi: I \rightarrow[0, \infty)$ are continuous such that every integral below exists. If a continuously differentiable function $y: I \rightarrow \mathbb{C}$ satisfies the differential inequality

$$
\left|y^{\prime}(x)+f(x) y(x)-g(x)\right| \leq \varphi(x)
$$

for all $x \in I$, then there exists a continuously differentiable function $y_{0}: I \rightarrow \mathbb{C}$ such that $y_{0}^{\prime}(x)+f(x) y_{0}(x)=$ $g(x)$ and

$$
\left|y(x)-y_{0}(x)\right| \leq \exp \left(\Re\left(\int_{x}^{c} f(w) d w\right)\right) \int_{x}^{c} \varphi(s) \exp \left(-\Re\left(\int_{s}^{c} f(w) d w\right)\right) d s
$$

for all $x \in I$.

\section{A Type of Hyers-Ulam Stability}

In this section, we assume that the potential function $V:(0, \infty) \rightarrow \mathbb{R}$ is a hyperbolic function defined by

$$
V(x)=V_{0}-\frac{\hbar^{2} \omega_{1}}{m} \cdot \frac{1}{x}
$$

where we set $\omega_{1}=\sqrt{\frac{2 m\left(V_{0}-E\right)}{\hbar^{2}}}$ and $0<E<V_{0}$.

For this potential function shown in Figure 1, we investigate a type of Hyers-Ulam stability of one-dimensional Schrödinger Equation (2) only in finite intervals such as $(0, c)$ because of the condition in Lemma 2 imposed on the coefficient of the differential equation. Even so, in this paper, there is great interest in the properties of solutions of the Schrödinger equation. In particular, we have a greater interest in their behaviors in the vicinity of $x=0$.

From now on, suppose that $c$ is a positive real number that satisfies $V(c)=V_{0}-\frac{\hbar^{2} \omega_{1}}{c m}>E$ unless specified otherwise. Using this $c$, we define the open interval $I=(0, c)$.

The following formulas define the differential operators $\mathcal{D}_{1}$ and $\mathcal{D}_{2}$ according to the potential function $V$ given in (3):

$$
\begin{aligned}
& \left(\mathcal{D}_{1} \psi\right)(x)=\psi^{\prime}(x)+\left(\omega_{1}-\frac{1}{x}\right) \psi(x), \\
& \left(\mathcal{D}_{2} \psi\right)(x)=\psi^{\prime}(x)+\left(\frac{1}{x}-\omega_{1}\right) \psi(x)
\end{aligned}
$$

where $\psi: I \rightarrow \mathbb{C}$ is any continuously differentiable function. 


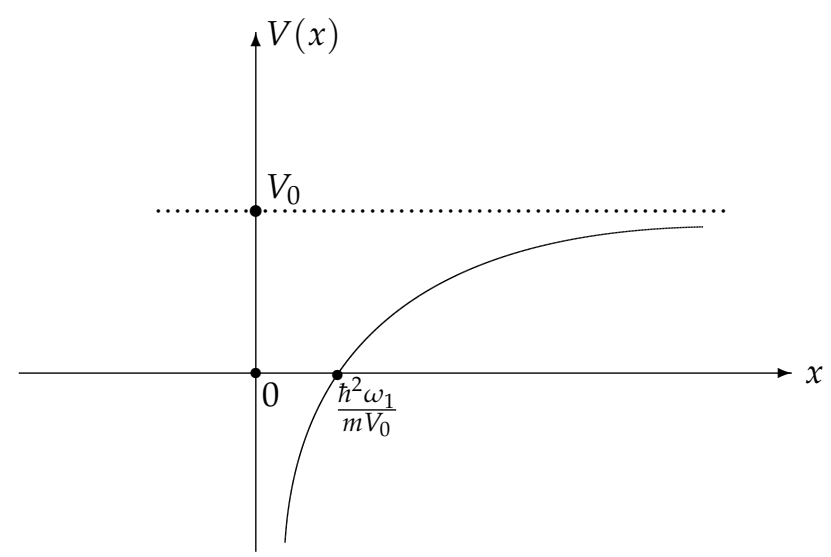

Figure 1. The hyperbolic potential well $V(x)$.

With those differential operators, we have

$$
\begin{aligned}
-\frac{\hbar^{2}}{2 m}\left(\left(\mathcal{D}_{2} \circ \mathcal{D}_{1}\right) \psi\right)(x) & =-\frac{\hbar^{2}}{2 m}\left(\psi^{\prime \prime}(x)+\left(\frac{2 \omega_{1}}{x}-\omega_{1}^{2}\right) \psi(x)\right) \\
& =-\frac{\hbar^{2}}{2 m} \psi^{\prime \prime}(x)+V(x) \psi(x)-E \psi(x) \quad(x \in I)
\end{aligned}
$$

for any twice continuously differentiable function $\psi: I \rightarrow \mathbb{C}$, which implies that

$$
\left|-\frac{\hbar^{2}}{2 m} \psi^{\prime \prime}(x)+V(x) \psi(x)-E \psi(x)\right| \leq \varepsilon \quad(x \in I)
$$

if and only if

$$
\left|\left(\left(\mathcal{D}_{2} \circ \mathcal{D}_{1}\right) \psi\right)(x)\right| \leq \frac{2 m}{\hbar^{2}} \varepsilon \quad(x \in I)
$$

which is equivalent to

$$
\left|\phi^{\prime}(x)+\left(\frac{1}{x}-\omega_{1}\right) \phi(x)\right| \leq \frac{2 m}{\hbar^{2}} \varepsilon \quad(x \in I),
$$

where we set $\phi(x)=\left(\mathcal{D}_{1} \psi\right)(x)$.

As we shall see in (6) below, the inequality $\left|\psi(x)-\psi_{0}(x)\right| \leq K_{\varepsilon}(x)$ holds true for any $x \in I$ and $\varepsilon>0$, where $K_{\varepsilon}(x)$ is strongly affected by values of $x$. Hence, the phenomenon observed in the following theorem is called a type of Hyers-Ulam stability.

Theorem 1. Assume that $E$ and $V_{0}$ are real numbers with $0<E<V_{0}$ and $V:(0, \infty) \rightarrow \mathbb{R}$ is the hyperbolic potential function defined by (3). Moreover, suppose that $c>0$ is a real number satisfying $V(c)=V_{0}-\frac{\hbar^{2} \omega_{1}}{c m}>E$ and $I=(0, c)$ is an open interval. Given any $\varepsilon>0$, if a twice continuously differentiable function $\psi: I \rightarrow \mathbb{C}$ satisfies the inequality

$$
\left|-\frac{\hbar^{2}}{2 m} \frac{d^{2} \psi(x)}{d x^{2}}+V(x) \psi(x)-E \psi(x)\right| \leq \varepsilon
$$


for all $x \in I$, then the one-dimensional time independent Schrödinger Equation (2) has a twice continuously differentiable solution $\psi_{0}: I \rightarrow \mathbb{C}$ with

$$
\begin{aligned}
& \left|\psi(x)-\psi_{0}(x)\right| \\
& \quad \leq \frac{2 m}{\hbar^{2} \omega_{1}} \varepsilon x e^{-\omega_{1} x}\left(\int_{x}^{c} \frac{1}{s} e^{\omega_{1} s} d s+\frac{1}{\omega_{1}} \int_{x}^{c} \frac{1}{s^{2}} e^{\omega_{1} s} d s-\left(c+\frac{1}{\omega_{1}}\right) e^{-\omega_{1} c} \int_{x}^{c} \frac{1}{s^{2}} e^{2 \omega_{1} s} d s\right)
\end{aligned}
$$

for all $x \in I$.

Proof. First, we define $\phi(x)=\left(\mathcal{D}_{1} \psi\right)(x)=\psi^{\prime}(x)+\left(\omega_{1}-\frac{1}{x}\right) \psi(x)$. On account of (4) and (5), Lemma 2 may apply to inequality (4) with reference to Table 1 :

Table 1. Substitutions.

\begin{tabular}{cc}
\hline Lemma 2 & In (4) \\
\hline$y(x)$ & $\phi(x)$ \\
$f(x)$ & $\frac{1}{x}-\omega_{1}$ \\
$g(x)$ & 0 \\
$\varphi(x)$ & $\frac{2 m}{\hbar^{2}} \varepsilon$ \\
\hline
\end{tabular}

Additionally, there exists a continuously differentiable function $\phi_{0}: I \rightarrow \mathbb{C}$ that satisfies

$$
\phi_{0}^{\prime}(x)+\left(\frac{1}{x}-\omega_{1}\right) \phi_{0}(x)=0
$$

as well as

$$
\begin{aligned}
\left|\phi(x)-\phi_{0}(x)\right| & \leq \frac{2 m}{\hbar^{2}} \varepsilon \exp \left(\int_{x}^{c}\left(\frac{1}{w}-\omega_{1}\right) d w\right) \int_{x}^{c} \exp \left(-\int_{s}^{c}\left(\frac{1}{w}-\omega_{1}\right) d w\right) d s \\
& =\frac{2 m}{\hbar^{2}} \varepsilon \frac{e^{\omega_{1} x}}{x}\left(\frac{1}{\omega_{1}}\left(x+\frac{1}{\omega_{1}}\right) e^{-\omega_{1} x}-\frac{1}{\omega_{1}}\left(c+\frac{1}{\omega_{1}}\right) e^{-\omega_{1} c}\right)
\end{aligned}
$$

for all $x \in I$.

Since we defined $\phi(x)=\psi^{\prime}(x)+\left(\omega_{1}-\frac{1}{x}\right) \psi(x)$, we see by (8) that

$$
\begin{aligned}
& \left|\psi^{\prime}(x)+\left(\omega_{1}-\frac{1}{x}\right) \psi(x)-\phi_{0}(x)\right| \\
& \quad \leq \frac{2 m}{\hbar^{2} \omega_{1}} \varepsilon\left(1+\frac{1}{\omega_{1}} \cdot \frac{1}{x}-\left(c+\frac{1}{\omega_{1}}\right) \frac{e^{-\omega_{1}(c-x)}}{x}\right)
\end{aligned}
$$

for all $x \in I$. Lemma 2 may again apply to inequality (9) with reference to Table 2:

Table 2. Substitutions.

\begin{tabular}{cc}
\hline Lemma 2 & In (9) \\
\hline$y(x)$ & $\psi(x)$ \\
$f(x)$ & $\omega_{1}-\frac{1}{x}$ \\
$g(x)$ & $\phi_{0}(x)$ \\
$\varphi(x)$ & $\frac{2 m}{\hbar^{2} \omega_{1}} \varepsilon\left(1+\frac{1}{\omega_{1}} \frac{1}{x}-\left(c+\frac{1}{\omega_{1}}\right) \frac{e^{-\omega_{1}(c-x)}}{x}\right)$ \\
\hline
\end{tabular}

On account of Lemma 2, there exists a continuously differentiable function $\psi_{0}: I \rightarrow \mathbb{C}$ that satisfies

$$
\psi_{0}^{\prime}(x)+\left(\omega_{1}-\frac{1}{x}\right) \psi_{0}(x)=\phi_{0}(x)
$$


as well as

$$
\begin{aligned}
\left|\psi(x)-\psi_{0}(x)\right| \\
\leq \frac{2 m}{\hbar^{2} \omega_{1}} \varepsilon \exp \left(\int_{x}^{c}\left(\omega_{1}-\frac{1}{w}\right) d w\right) . \\
\quad \cdot \int_{x}^{c}\left(1+\frac{1}{\omega_{1}} \frac{1}{s}-\left(c+\frac{1}{\omega_{1}}\right) \frac{e^{-\omega_{1}(c-s)}}{s}\right) \exp \left(-\int_{s}^{c}\left(\omega_{1}-\frac{1}{w}\right) d w\right) d s \\
=\frac{2 m}{\hbar^{2} \omega_{1}} \varepsilon x e^{-\omega_{1} x}\left(\int_{x}^{c} \frac{1}{s} e^{\omega_{1} s} d s+\frac{1}{\omega_{1}} \int_{x}^{c} \frac{1}{s^{2}} e^{\omega_{1} s} d s-\left(c+\frac{1}{\omega_{1}}\right) e^{-\omega_{1} c} \int_{x}^{c} \frac{1}{s^{2}} e^{2 \omega_{1} s} d s\right)
\end{aligned}
$$

for all $x \in I$.

Moreover, by using Lemma 1 and Equation (10), we show that $\psi_{0}$ has the form

$$
\psi_{0}(x)=\exp \left(\int_{x}^{c}\left(\omega_{1}-\frac{1}{w}\right) d w\right)\left(\psi_{0}(c)-\int_{x}^{c} \phi_{0}(s) \exp \left(-\int_{s}^{c}\left(\omega_{1}-\frac{1}{w}\right) d w\right) d s\right),
$$

where $\psi_{0}(c)$ is assumed to be an arbitrary complex number. Furthermore, twice continuous differentiability of $\psi_{0}$ follows from the continuous differentiability of $\phi_{0}$.

By using (7) and (10), we can finally verify that $\psi_{0}: I \rightarrow \mathbb{C}$ is a solution to the one-dimensional, time independent Schrödinger Equation (2).

If the value of $x$ is very close to 0 , we can calculate as follows:

$$
\begin{aligned}
\frac{1}{x} e^{\omega_{1} x} & =\frac{1}{x}+\sum_{n=1}^{\infty} \frac{\omega_{1}^{n}}{n !} x^{n-1}, \\
\frac{1}{x^{2}} e^{\omega_{1} x} & =\frac{1}{x^{2}}+\frac{\omega_{1}}{x}+\sum_{n=2}^{\infty} \frac{\omega_{1}^{n}}{n !} x^{n-2}, \\
\frac{1}{x^{2}} e^{2 \omega_{1} x} & =\frac{1}{x^{2}}+\frac{2 \omega_{1}}{x}+\sum_{n=2}^{\infty} \frac{\left(2 \omega_{1}\right)^{n}}{n !} x^{n-2}
\end{aligned}
$$

and

$$
\begin{aligned}
& x \int_{x}^{c} \frac{1}{s} e^{\omega_{1} s} d s=x \ln c-x \ln x+x \sum_{n=1}^{\infty} \frac{\omega_{1}^{n}}{n ! n}\left(c^{n}-x^{n}\right), \\
& x \int_{x}^{c} \frac{1}{s^{2}} e^{\omega_{1} s} d s=1-\frac{x}{c}+\omega_{1} x(\ln c-\ln x)+x \sum_{n=2}^{\infty} \frac{\omega_{1}^{n}}{n !(n-1)}\left(c^{n-1}-x^{n-1}\right), \\
& x \int_{x}^{c} \frac{1}{s^{2}} e^{2 \omega_{1} s} d s=1-\frac{x}{c}+2 \omega_{1} x(\ln c-\ln x)+x \sum_{n=2}^{\infty} \frac{\left(2 \omega_{1}\right)^{n}}{n !(n-1)}\left(c^{n-1}-x^{n-1}\right) .
\end{aligned}
$$

Hence, we get

$$
x \int_{x}^{c} \frac{1}{s} e^{\omega_{1} s} d s=O(x), \quad x \int_{x}^{c} \frac{1}{s^{2}} e^{\omega_{1} s} d s=O(1), \quad x \int_{x}^{c} \frac{1}{s^{2}} e^{2 \omega_{1} s} d s=O(1)
$$

as $x \rightarrow 0^{+}$, where $O(\cdot)$ denotes the Big O notation.

As already mentioned earlier in this section, we have a great interest in the approximation property (in the vicinity of $x=0$ ) of the solutions of the time independent Schrödinger equation. Therefore the following corollary will be very interesting to us.

Corollary 1. Assume that $E$ and $V_{0}$ are real numbers with $0<E<V_{0}$ and $V:(0, \infty) \rightarrow \mathbb{R}$ is the hyperbolic potential function defined by (3). Moreover, suppose that $c>0$ is a real number satisfying $V(c)=V_{0}-\frac{\hbar^{2} \omega_{1}}{c m}>E$ and $I=(0, c)$ is an open interval. Given any $\varepsilon>0$, if a twice continuously 
differentiable function $\psi: I \rightarrow \mathbb{C}$ satisfies the inequality (5) for any $x \in I$, then the one-dimensional, time independent Schrödinger Equation (2) has a twice continuously differentiable solution $\psi_{0}: I \rightarrow \mathbb{C}$ with

$$
\left|\psi(x)-\psi_{0}(x)\right|=O(1)
$$

as $x \rightarrow 0^{+}$. In particular, there exists a real constant $K>0$ such that

$$
\left|\psi(x)-\psi_{0}(x)\right| \leq K \varepsilon
$$

for all sufficiently small values of $x>0$.

\section{Discussion}

The Schrödinger equation is a linear partial differential equation that describes the wave function of the quantum-mechanical system and this equation plays a key role in quantum mechanics.

The form of the Schrödinger equation looks different depending on the physical situation. The most general form is the time dependent Schrödinger equation, which describes the state of a system that evolves with time. Among the predictions of the time dependent Schrödinger equation is that the wave functions can form standing waves (stationary states). Stationary states are especially important because their individual states simplify the task of solving the time dependent Schrödinger equation. The stationary state can be well explained by the time independent Schrödinger equation, which is the simpler form of the Schrödinger equation.

The above descriptions ensure well that the subject of our paper is of considerable importance.

Before we can prove the "exact" Hyers-Ulam stability of the Schrödinger Equation (2), wherein a hyperbolic potential function works, we will need to further improve Lemma 2. However, we do not think it is realistic to significantly improve Lemma 2 in this small paper. Therefore, we think it is wiser to leave this improvement as an open problem rather than to seek it in this paper.

\section{Conclusions}

Only when the potential function is exactly expressed in the form of (3) do we cautiously predict that we will be able to demonstrate a type of Hyers-Ulam stability of the one-dimensional, time independent Schrödinger equation by using the operator method.

It would be more general to prove the stability of the Schrödinger equation under the action of the potential function $V(x)=\alpha+\frac{\beta}{x}$-where $\alpha$ and $\beta$ meet only the minimum requirements, and if possible they are independent of each other-but unfortunately in this paper we did not. From this point of view, the main result of this paper seems a little awkward. Nevertheless, it is a great advantage of this paper that we have demonstrated a type of Hyers-Ulam stability of Schrödinger's equation under the action of the potential function that has a singularity point.

Author Contributions: Conceptualization, G.C. and S.-M.J.; writing-original draft preparation, G.C. and S.-M.J.; writing-review and editing, G.C. and S.-M.J.; funding acquisition, G.C. and S.-M.J. All authors have read and agreed to the published version of the manuscript.

Funding: This work was supported by the National Research Foundation of Korea (NRF) grant funded by the Korea government (MSIT) (number 2020R1F1A1A01049560). This work was supported by 2020 Hongik University Research Fund.

Conflicts of Interest: The authors declare no conflict of interest.

\section{References}

1. Ulam, S.M. A Collection of Mathematical Problems; Interscience Publishers: New York, NY, USA, 1960.

2. Hyers, D.H. On the stability of the linear functional equation. Proc. Natl. Acad. Sci. USA 1941, 27, $222-224$. [CrossRef] [PubMed] 
3. Rassias, T.M. On the stability of the linear mapping in Banach spaces. Proc. Am. Math. Soc. 1987, 72, $297-300$. [CrossRef]

4. Găvruţa, P. A generalization of the Hyers-Ulam-Rassias stability of approximately additive mappings. J. Math. Anal. Appl. 1994, 184, 431-436. [CrossRef]

5. Hyers, D.H.; Isac, G.; Rassias, T.M. Stability of Functional Equations in Several Variables; Birkhäuser: Boston, MA, USA, 1998.

6. Jung, S.-M. Hyers-Ulam-Rassias Stability of Functional Equations in Nonlinear Analysis; Springer Optimization and It's Applications; Springer: New York, NY, USA, 2011; Volume 48.

7. Sahoo, P.K.; Kannappan, P.L. Introduction to Functional Equations; CRC Press: Boca Raton, FL, USA, 2011.

8. Obłoza, M. Hyers stability of the linear differential equation. Rocznik Nauk.-Dydakt. Prace Mat. 1993, 13, 259-270.

9. Obłoza, M. Connections between Hyers and Lyapunov stability of the ordinary differential equations. Rocznik Nauk.-Dydakt. Prace Mat. 1997, 14, 141-146.

10. Alsina, C.; Ger, R. On some inequalities and stability results related to the exponential function. J. Inequal. Appl. 1998, 2, 373-380. [CrossRef]

11. Choi, G.; Jung, S.-M. Invariance of Hyers-Ulam stability of linear differential equations and its applications. Adv. Differ. Equ. 2015, 277, 1-14. [CrossRef]

12. Choi, G.; Jung, S.-M.; Roh, J. Some properties of approximate solutions of linear differential equations. Mathematics 2019, 7, 806. [CrossRef]

13. Cîmpean, D.S.; Popa, D. On the stability of the linear differentia equation of higher order with constant coefficients. Appl. Math. Comput. 2010, 217, 4141-4146.

14. Jung, S.-M. Hyers-Ulam stability of linear differential equations of first order. Appl. Math. Lett. 2004, 17, 1135-1140. [CrossRef]

15. Jung, S.-M. Hyers-Ulam stability of linear differential equations of first order, II. Appl. Math. Lett. 2006, 19, 854-858. [CrossRef]

16. Jung, S.-M.; Roh, J. Hyers-Ulam stability of the time independent Schrödinger equations. Appl. Math. Lett. 2017, 74, 147-153. [CrossRef]

17. Li, Y.; Shen, Y. Hyers-Ulam stability of linear differential equations of second order. Appl. Math. Lett. 2010, 23, 306-309. [CrossRef]

18. Popa, D.; Raşa, I. On the Hyers-Ulam stability of the linear differential equation. J. Math. Anal. Appl. 2011, 381, 530-537. [CrossRef]

19. Takahasi, S.-E.; Miura, T.; Miyajima, S. On the Hyers-Ulam stability of the Banach space-valued differential equation $y^{\prime}=\lambda y$. Bull. Korean Math. Soc. 2002, 39, 309-315. [CrossRef]

20. Wang, G.; Zhou, M.; Sun, L. Hyers-Ulam stability of linear differential equations of first order. Appl. Math. Lett. 2008, 21, 1024-1028. [CrossRef]

21. Jung, S.-M.; Kim, B. Perturbation of one-dimensional time independent Schrödinger equation with a symmetric parabolic potential wall. Symmetry 2020, 12, 1089. [CrossRef]

22. Choi, G.; Jung, S.-M.; Roh, J. An operator method for the stability of inhomogeneous wave equations. Symmetry 2019, 11, 324. [CrossRef]

(C) 2020 by the authors. Licensee MDPI, Basel, Switzerland. This article is an open access article distributed under the terms and conditions of the Creative Commons Attribution (CC BY) license (http:/ / creativecommons.org/licenses/by/4.0/). 